\title{
A meta-analysis of metacognitive beliefs as implicated in the self-regulatory executive function model in clinical psychosis
}

DOI:

10.1016/j.schres.2016.09.032

\section{Document Version}

Accepted author manuscript

Link to publication record in Manchester Research Explorer

Citation for published version (APA):

Sellers, R., Varese, F., Wells, A., \& Morrison, A. (2016). A meta-analysis of metacognitive beliefs as implicated in the self-regulatory executive function model in clinical psychosis. Schizophrenia Research.

https://doi.org/10.1016/j.schres.2016.09.032

\section{Published in:}

Schizophrenia Research

\section{Citing this paper}

Please note that where the full-text provided on Manchester Research Explorer is the Author Accepted Manuscript or Proof version this may differ from the final Published version. If citing, it is advised that you check and use the publisher's definitive version.

\section{General rights}

Copyright and moral rights for the publications made accessible in the Research Explorer are retained by the authors and/or other copyright owners and it is a condition of accessing publications that users recognise and abide by the legal requirements associated with these rights.

\section{Takedown policy}

If you believe that this document breaches copyright please refer to the University of Manchester's Takedown Procedures [http://man.ac.uk/04Y6Bo] or contact uml.scholarlycommunications@manchester.ac.uk providing relevant details, so we can investigate your claim.

\section{OPEN ACCESS}


A meta-analysis of metacognitive beliefs as implicated in the Self-Regulatory Executive Function model in clinical psychosis

Rachel Sellers ${ }^{\mathrm{ab}}$

Filippo Varese ${ }^{a}$

Adrian Wells ${ }^{\mathrm{a}}$

Anthony P. Morrison ${ }^{\mathrm{ab}}$

${ }^{\text {a }}$ School of Psychological Sciences, University of Manchester, Oxford Road, Manchester, M13 9PL

${ }^{\mathrm{b}}$ Psychosis Research Unit, Greater Manchester West Mental Health Foundation NHS Trust, Bury New Road, Prestwich, Manchester, M25 3BL

Corresponding author: Rachel Sellers, Psychosis Research Unit, Greater Manchester West Mental Health Foundation NHS Trust, Bury New Road, Prestwich, Manchester, M25 3BL

Email: Rachel.sellers@gmw.nhs.uk; Telephone: 0161358 1395; Fax: 0161772 4351 


\section{Abstract}

This meta-analysis investigated whether the five metacognitive beliefs implicated in the Self-Regulatory Executive Function (S-REF) model (Wells \& Matthews, 1994; Wells \& Matthews, 1996) are elevated in people with clinical psychosis compared to people with emotional disorder and non-psychiatric controls. The review followed guidance set-out in the PRISMA statement. Primary analyses compared summary effect sizes on each sub-scale of the Metacognitions Questionnaire (MCQ) for people with psychosis and non-psychiatric controls; and people with psychosis and people with emotional disorder. Eleven eligible studies were identified comprised of 568 psychosis participants, 212 emotional disorder participants and 776 nonpsychiatric controls. Findings indicated that people with psychosis had higher scores on all sub-scales of the MCQ compared to non-psychiatric controls; and higher scores on the positive beliefs about worry sub-scale compared to people with emotional disorder. This suggests metacognitive beliefs may be associated with the presence of psychological disorder and distress in general, rather than specific diagnoses. Implications for models of psychosis and treatment are discussed. Key words: Metacognition; psychosis; negative affect 
1. Introduction

In recent years there has been an increase in research investigating metacognition in psychosis. The term metacognition describes "thinking about thinking" (Papaleontiou-Louca, 2003) and refers to "any knowledge or cognitive process that is involved in the appraisal, monitoring or control of cognition" (Wells, 2000, p. 6). Within the psychosis literature, metacognition has been investigated in different ways. Lysaker and colleagues use of the term metacognition reflects an individual's ability to form and understand complex representations about themselves, others, and the world (Lysaker et al., 2010). Moritz and colleagues have carried out numerous studies investigating metacognitive training that focuses on cognitive biases associated with attributional style and jumping to conclusions (Moritz, Vitzthum, Randjbar, Veckenstedt, \& Woodward, 2010). There have been previous reviews relating to these definitions (Lysaker et al., 2013; van Oosterhout et al., 2016). Another influential line of research has focused on the metacognitive factors implicated in the Wells and Matthews Self-Regulatory Executive Function (S-REF) model (Wells \& Mattews, 1994; Wells \& Matthews, 1996). This approach emphasises metacognitive beliefs and strategies that are proposed to maintain unhelpful thinking styles and distress across disorders, and there is accumulating evidence that the metacognitive beliefs implicated in this model may be related to distressing experiences of psychosis. Currently, the specific nature of this relationship requires further clarification.

The S-REF model (Wells \& Matthews, 1996) focuses on unhelpful metacognitive beliefs and emotional self-regulation strategies that lead to biased information processing of threatening stimuli. Such strategies include enhanced verbal processing in the form of worry and rumination, attentional biases in the form of 
threat monitoring, and attempts to control thoughts and other internal events. These responses constitute a style of thinking known as The Cognitive Attentional Syndrome (CAS) that is proposed to maintain unhelpful thinking patterns and distress. The CAS arises from unhelpful metacognitive beliefs that are positive and negative in content. Positive metacognitive beliefs reflect the usefulness of worry, rumination, threat monitoring, and other similar strategies (Wells, 2009). They include beliefs such as "focussing on danger will keep me safe" or "if I worry I will be prepared" and promote the implementation of unhelpful coping responses. Negative metacognitive beliefs reflect beliefs concerning the danger or uncontrollability of particular thoughts and affect how thoughts and thought processes are appraised (Wells, 2009). They include beliefs such as "thoughts can make bad things happen" or "my worrying is uncontrollable". The co-occurrence of positive and negative metacognitive beliefs is thought to be related to greater pathology (Wells, 2000).

Consistent with these assumptions, research has found evidence of a positive relationship between unhelpful metacognitive beliefs and emotional disorder. A greater endorsement of negative metacognitive beliefs has been associated with anxiety (Davis \& Valentier, 2000; McEvoy \& Mahoney, 2013), depression (Papageorgiou \& Wells, 2001) and obsessive compulsive disorder (Wells \& Papageorgiou, 1998). In addition prospective cohort studies have found that higher levels of unhelpful metacognitive beliefs at baseline predict subsequent severity of anxiety and depression (Hjemdal, Stiles, \& Wells, 2013; Papageorgiou \& Wells, 2009; Yilmaz, Gencoz, \& Wells, 2011). Metacognitive beliefs have also been found to mediate relationships between symptoms and distress (Dragan \& Dragan, 2014; Irak \& Tosun, 2008). 
The application of the metacognitive model to psychosis has also received support from cross-sectional and cohort studies. For example, research using non-clinical samples suggests that people with higher proneness to hallucinations and delusions tend to have an increased number of both positive and negative metacognitive beliefs (Laroi \& Van der Linden, 2005; Morrison, Wells, \& Nothard, 2000). Research using samples of people at risk of developing psychosis suggests that at risk mental state (Morrison et al., 2006) and subsequent transition to first episode psychosis (Barbato et al., 2013; Morrison, Bentall, et al., 2002) is associated with a greater endorsement of negative metacognitive beliefs. A higher number of negative beliefs has also been related to increased distress (Barbato et al., 2013; Brett, Johns, Peters, \& McGuire, 2009; Oosterhout, Krabbendam, Smeets, \& van der Gaag, 2013) and a more severe and chronic course of illness (Austin et al., 2015).

Currently, the specific role of metacognitive beliefs in psychosis is unclear. A previous meta-analysis (Varese \& Bentall, 2011) found limited evidence that metacognitive beliefs have a causal role in specific symptoms of psychosis (i.e. auditory hallucinations) as previous models suggested (Morrison, Haddock, \& Tarrier, 1995). Instead there is emerging evidence that metacognitive beliefs may be a general vulnerability factor to psychological disorder and that metacognitive beliefs (and associated CAS activity) may influence symptom maintenance, help-seeking and distress (Hill, Varese, Jackson, \& Linden, 2012; Varese, Barkus, \& Bentall, 2011).

To test the prediction that metacognitive beliefs are associated with psychological disorder and unhelpful thinking styles in general rather than specific diagnoses, this meta-analysis will use quantitative methods to compare levels of unhelpful metacognitive beliefs in people with clinical psychosis, people with emotional 
disorder and people with no psychiatric diagnosis. The following research questions will be addressed: (i) Do people with psychosis have elevated levels of unhelpful metacognitive beliefs compared to non-psychiatric controls? (ii) Do people with psychosis have elevated levels of unhelpful metacognitive beliefs compared to people with emotional disorder?

\section{Method}

The review followed guidance set out in the Preferred Reporting Items for Systematic reviews and Meta-Analyses (Moher, Liberati, Tetzlaff, \& Altman, 2009).

\subsection{Operationalization of concepts}

To minimise ambiguity in study inclusion the following operationalization of key concepts were used:

Psychosis: A diagnosis of psychotic disorder according to the Diagnostic Statistical Manual of Mental Disorders fourth edition (American Psychiatric Association, 2000) or International Classification of Diseases tenth edition (World Health Organisation, 1993); or meets threshold for early intervention in psychosis using the Positive and Negative Syndrome Scale (Kay, Fiszbein, \& Opler, 1987) defined as a score of four on hallucinations or delusional beliefs or a score of five on paranoid ideation. People with a diagnosis of Bipolar Disorder were not considered eligible.

Emotional Disorder: A diagnosis of a depression or anxiety disorder (such as panic disorder, generalised anxiety disorder, obsessive compulsive disorder) according to the Diagnostic Statistical Manual of Mental Disorders fourth edition (American Psychiatric Association, 2000) or International Classification of Diseases 
tenth edition (World Health Organisation, 1993). The emotional disorder groups were extracted from eligible studies that included a psychosis sample.

Non-psychiatric controls: No formal diagnosis of a psychiatric disorder. The non-psychiatric controls were extracted from eligible studies that included a psychosis sample.

Metacognitive beliefs: Metacognitive beliefs were restricted to those captured by the Meta-Cognitions Questionnaire (MCQ) and its variants (Cartwright-Hatton \& Wells, 1997; Wells \& Cartwright-Hatton, 2004). The MCQ was designed specifically to measure the five metacognitive beliefs implicated in the S-REF model. Previous studies have identified alternative measures of metacognitive beliefs specific to experiences of psychosis, such as the Beliefs about Paranoia Scale (Morrison, 2005) and the Interpretation of Voices Inventory (Morrison, Wells, \& Nothard, 2002). However, given that the present review aims to quantify the magnitude of the relationship between those beliefs implicated in emotional regulation across a range of psychological disorders, metacognitive beliefs were restricted to those central to this model. In addition, these alternative measures may be less relevant to our control groups.

The MCQ-65 (Cartwright-Hatton \& Wells, 1997) and MCQ-30 (Wells \& CartwrightHatton, 2004) yield five sub-scales consisting of "positive beliefs about worry" reflecting the belief that worry can help to solve problems; "negative beliefs including the uncontrollability and danger of thoughts" reflecting the belief that thoughts must be controlled in order to function well; "cognitive confidence" capturing the extent to which an individual has confidence in their memory and attentional capabilities; "negative beliefs including responsibility and superstition" reflecting superstitious 
themes that certain thoughts can cause negative outcomes, and feelings of responsibility for preventing these outcomes; and "cognitive self-consciousness" reflecting the extent to which an individual engages in monitoring their own thought processes. Higher scores on each sub-scale indicate a greater endorsement of unhelpful beliefs. The MCQ-SAM (Lobban, 1998) is a modified and shortened version of the MCQ-65 that contains two additional sub-scales. Factor analysis indicates that the first four sub-scales reliably capture positive beliefs about worry, negative beliefs including uncontrollability and danger, cognitive confidence and cognitive self-consciousness. The two remaining sub-scales of the MCQ-SAM will be excluded from analyses because they do not form part of the S-REF model.

\subsection{Search strategy}

A comprehensive and systematic review of the literature was carried out in three stages. First, studies were identified by searching PsychInfo, PubMed and EMBASE. Medical Subject Headings (MeSH) "psychosis" and "metacognition" were supplemented with text word searches (psychos* or psychoti ${ }^{*}$ or schizo* or paranoi* or delu* or hallucinat*) and (metacog* or self-focus" or "cognitive attentional syndrome" or worry or ruminat* or "thought suppress" or "thought control" or "metaworry") and combined. Second, an inspection of eligible study reference lists was carried out to identify any relevant studies missed through database searching (forward and backward tracking). Finally, citations of the original validation papers of the MCQ-30 and MCQ-65 were identified using SCOPUS and cross-checked against our database search results. Searches were updated and completed in September 2015.

\subsection{Eligibility screening}


Studies were eligible for the meta-analysis if (i) the study investigated the relationship between metacognitive beliefs and psychosis; (ii) participants met diagnostic or early intervention criteria for a psychotic disorder; (iii) the study utilised a comparison group of people with a diagnosis of emotional disorder or nonpsychiatric controls; (iv) the study contained sufficient statistical information for extraction or sufficient data could be retrieved from authors. Studies were not included in the review if they were not reported in English. Eligibility was assessed in a three stage procedure by the first author (first by title, then by abstract and finally by full text). Ambiguities in study eligibility were resolved by consultation with the senior authors.

\subsection{Methodological quality}

Methodological quality of included studies was assessed independently by two raters using a tool for assessing the quality of observational studies. Disagreements in quality ratings were resolved through consultation with the senior authors. This tool has been adapted from the Agency for Healthcare Research and Quality (Williams, Plassman, Burke, Holsinger, \& Benjamin, 2010) and has previously been applied to assessing methodological quality of observational studies for people with psychosis at risk of self-harm and suicide (Taylor, Hutton, \& Wood, 2015). The tool uses a four point rating system (meets, partially meets, does not meet or unclear) to grade ten criteria concerned with the methods used to select the sample, the adequacy of the sample size, the methods used to ascertain non-clinical/clinical status and the appropriateness of the statistical analyses.

\subsection{Data extraction}


Data extraction was carried out by the first author following a pre-defined coding protocol agreed by all four authors. The extraction of study characteristics included the sample characteristics, the research design, the instruments used to assess symptoms, and the instruments used to assess metacognitive beliefs. The extraction of study data included the sample means and standard deviations reported for psychosis groups, emotional disorder groups and non-psychiatric control groups on each sub-scale of the MCQ. When such descriptive statistics were not available authors were contacted for this information.

\subsection{Effect size calculations and data analysis}

All analyses were carried out using Comprehensive Meta-analysis version 3. Hedges' $g$ was selected as the effect size metric for the analyses, and was calculated using sample means, standard deviations and sample sizes extracted from eligible studies. All analyses were carried out under the random-effects model. This was selected due to the likelihood of methodological and sampling variation in the different cross-sectional studies included. The random effects model assumes there is no common effect for all included studies and that the included studies/effect sizes represent a random sample from a larger population of studies/effect sizes (Liberati et al., 2009).

Two sets of effects (and associated variances) were calculated based on the statistical information extracted from the primary studies. To examine the first research question (i.e. whether people with psychosis have elevated levels of unhelpful metacognitive beliefs compared to non-psychiatric controls), standardised mean difference effects were calculated for comparisons between psychosis participant's vs non-psychiatric controls. To examine the second research question 
(i.e. whether people with psychosis have elevated levels of unhelpful metacognitive beliefs compared to people with emotional disorder), standardised mean difference

effects were calculated for comparisons between psychosis participants vs emotional disorder participants on the five MCQ sub-scales. Where separate means were reported for sub-groups of psychosis within a study (e.g. those people with auditory hallucinations and those without auditory hallucinations) a combined effect size was calculated (Borenstein, Hedges, Higgins, \& Rothstein, 2011).

\section{Results}

\subsection{Literature search results}

A total of 2427 articles were retrieved through the search strategy; 249 duplicates were removed resulting in 2178 papers for screening. Eligibility was assessed in a three stage procedure (first by title, then by abstract and finally by full text). An overview of the screening procedure is provided in Figure 1. Fifteen articles were considered eligible for the analysis; four further articles were removed due to reporting secondary analyses from which the primary paper was included. The remaining eligible articles consisted of eleven clinical studies with a total of 568 psychosis participants, 212 emotional disorder participants (Adjustment Disorder= 8; Anxiety $=24$; Depression $=44$; Dysthymic Disorder $=4$; Generalised Anxiety Disorder $=3$; Obsessive-Compulsive Disorder $=78$; Panic Disorder $=51$ ) and 776 nonpsychiatric controls. Participants that did not meet PANSS criteria for early intervention in psychosis were excluded from the sample extracted from Hill et al. (2012). A summary of eligible studies can be found in table 1.

\section{INSERT FIGURE 1}




\section{INSERT TABLE 1}

\subsection{Methodological quality assessment and risk of bias}

The data utilised in this review was exclusively cross-sectional. Only few longitudinal studies have investigated these relationships to date (e.g. Austin et al. 2015; Morrison et al. 2002). Methodological quality has been assessed in this context.

Common methodological weaknesses related to limited detail about recruitment and sampling. Cross-sectional data extracted from clinical trials provided detailed information relating to inclusion criteria, recruitment and sample characteristics. However, approximately half of the studies provided limited descriptions of recruitment methods. In terms of sampling, studies employing non-psychiatric controls tended to rely on self-reporting rather than using a validated tool to screen for past or current psychiatric disorder. Reporting of sample characteristics was limited and generally restricted to age and gender without additional context (e.g. duration of illness, social-economic status, and ethnicity). These weaknesses could affect generalizability of findings. They also limit the extent to which confounding variables can be considered at study level. In terms of methodology, the majority of studies did not use assessor blinding or were unclear about whether blinding procedures were implemented. There was also a tendency for power calculations and missing data to be unreported. A summary of methodological quality of the included studies can be found in table 2 .

Due to the measurement of metacognitive beliefs being restricted to those implicated in the S-REF model there is a possible risk of bias relating to the source of study data. Four of the eleven studies included in the analysis include a primary author of the model being tested (Wells) and a further two studies include an author of this 
paper (Morrison or Varese). However, the remaining data included in this metaanalysis came from a diversity of research teams and from different countries (i.e. Australia, Denmark, Germany, Spain and the United Kingdom). The description of study weaknesses above and the summary table provided (table 2) indicate the majority of studies did not meet a number of important quality criteria suggesting all studies have been rated objectively. In addition, visual and statistical methods of publication bias were utilised.

\section{INSERT TABLE 2}

3.3 Do people with psychosis have elevated levels of unhelpful metacognitive beliefs compared to non-psychiatric controls?

The primary research question was addressed using a series of separate metaanalyses. Analyses compared the effect sizes of each MCQ sub-scale in psychosis to the effect sizes of each sub-scale in non-psychiatric controls $(k=11)$. Figure two displays the individual effect size estimates with the variance and $95 \%$ confidence intervals for each analysis.

The analyses revealed that scores on the five sub-scales of the MCQ are significantly elevated in psychosis compared to non-psychiatric controls: positive beliefs $(g=0.55$, variance $=0.01, \mathrm{Cl}=0.34-0.76, \mathrm{p}<.001)$; negative beliefs including uncontrollability and danger $(g=1.10$, variance $=0.03, \mathrm{Cl}=0.78-1.42, \mathrm{p}<.001)$; cognitive confidence $(g=0.83$, variance $=0.02, \mathrm{Cl}=0.54-1.12, \mathrm{p}<.001)$; negative beliefs including responsibility and superstition $(g=1.31$, variance $=0.03, \mathrm{Cl}=0.96$ $1.66, p<.001)$; and cognitive self-consciousness $(g=0.49$, variance $=0.10 ; C l=0.29$ $0.69, \mathrm{p}<.001)$. Cohen (1988) effect size criteria traditionally applies to effect size estimates of $d$, however, Hedges $g$ is a small adjustment of Cohen's $d$. It is therefore 
considered reasonable to apply the same conventions. Cohen suggests that an effect size of $\geq .20$ is small, $\geq .50$ is moderate and $\geq .80$ is large. Utilising these criteria, cognitive self-consciousness had a small effect size whilst positive beliefs had a moderate effect, and negative beliefs and lack of cognitive confidence demonstrated large effect sizes.

\section{INSERT FIGURE 2}

3.4 Do people with psychosis have elevated levels of unhelpful metacognitive beliefs compared to people with emotional disorder?

The second research question was analysed by comparing the effect size of each MCQ sub-scale in psychosis to the effect size of each sub-scale in emotional disorder $(k=7)$. Figure three displays the individual effect size estimates with the variance and $95 \%$ confidence intervals for each analysis.

The analyses revealed that positive beliefs about worry were significantly higher in psychosis $(\mathrm{g}=.39$, variance $=0.01, \mathrm{Cl}=0.16-0.61, \mathrm{p}=.001)$. According to Cohen (1988) this is a small effect size. All other effect sizes between the psychosis and emotional disorder groups failed to reach significance

\section{INSERT FIGURE 3}

\subsection{Heterogeneity analyses}

Heterogeneity was tested using the $Q$ statistic. The results suggest that there is significant heterogeneity in effect size estimates between psychosis and nonpsychiatric controls and psychosis and emotional disorder controls on all sub-scales of the MCQ. Table 3 displays a summary of these results. It should be noted that this test has limited statistical power for meta-analyses with a small number of 
studies (Higgins \& Thompson, 2002). However, if a less conservative $p$ value of 0.10 is utilised, only the psychosis vs. emotional disorder positive beliefs analysis does not show significant heterogeneity. This indicates that variation in the true effect sizes varies more than would be expected due to sampling error.

Inconsistency in effect sizes across studies was quantified using the $\mathrm{I}^{2}$ statistic. The $\mathrm{I}^{2}$ statistic provides a percentage of variability in point estimates that is due to "true" heterogeneity rather than sampling error (Higgins \& Thompson, 2002). Examination of $\mathrm{I}^{2}$ indicates that the majority of estimates of the true effect size in the primary analyses have moderate to high heterogeneity according to Higgins and Thompson (2002). However, the $\mathrm{I}^{2}$ statistic also suffers uncertainty when only few studies are available and should be interpreted with caution (Liberati et al., 2009).

\section{INSERT TABLE 3}

\subsection{Publication bias}

All primary analyses were tested for publication bias (or other selection bias). Publication bias was assessed through inspection of funnel plots asymmetry, using both visual and statistical (i.e. Egger's test) methods. Inspection of the funnel plots and Eggers test did not indicate the presence of significant publication bias.

\section{Discussion}

This review investigated the relationship between the five metacognitive beliefs implicated in the Self-Regulatory Executive Function (S-REF) model proposed by Wells and Matthews $(1994,1996)$ and clinical psychosis. Specifically, meta-analysis was used to test whether metacognitive beliefs are elevated in people with psychosis compared to people with emotional disorder and non-psychiatric controls. 
The findings indicated that compared with non-psychiatric controls those with psychosis had significantly elevated scores on all five subscales of the MetaCognitions Questionnaire (MCQ). When compared to people with emotional disorder those with psychosis showed significantly higher scores on positive beliefs about worry, but not the other MCQ subscales. Our findings suggest that the five metacognitive beliefs implicated in the S-REF model are elevated in people with psychosis compared to people without a diagnosis, and there appears to be a high level of similarity between people with psychosis and those with emotional disorders in their elevated scores. These results support the S-REF model which suggests a common set of metacognitive beliefs may be associated with a vulnerability to psychological disorder, emotional regulation and distress rather than specific diagnoses.

It is possible that our finding of a high level of similarity between psychosis and emotional disorder groups is inflated by comorbid factors. However, it is acknowledged that anxiety and depression commonly occur in people with psychosis and may have a direct influence in the content of symptoms such as delusional beliefs (see Freeman \& Garety, 2003). In addition, emotional factors appear to have a pivotal role in the transition from sub-clinical to clinical experiences of psychosis with apparent dose-response relationships (van Rossum, Dominguez, Lieb, Wittchen, \& van Os, 2011). This has led to the suggestion that anxiety and depression may be considered necessary precursors to the onset of a psychotic disorder (Dominguez, Wichers, Lieb, Wittchen, \& van Os, 2011). Therefore, the investigation of common factors associated with emotional dysregulation in psychosis and emotional disorders may have important implications for psychological models and the prevention and treatment of psychosis. Our findings 
support cognitive models that recognise the importance of pre-morbid and cooccurring emotional factors (e.g. Garety, Kuipers, Fowler, Freeman, \& Bebbington, 2001; Morrison, 2001).

Our findings appeared to show some additional influence of positive metacognitive beliefs in psychosis when compared with emotional disorder. Positive metacognitive beliefs are concerned with the advantages of engaging in enhanced cognitive processing, in particular, they reflect beliefs about the usefulness or worry, rumination and threat monitoring. This may be an important finding if replicated as strong positive beliefs are likely to underlie particularly inflexible variants of the Cognitive Attentional Syndrome (i.e. repetitive negative thinking). The combination of such beliefs with beliefs about the uncontrollability of thoughts is likely to be especially problematic for attempts to disengage from perseverative forms of processing. However, this requires further investigation.

\section{Clinical implications}

The findings of the present review suggest that unhelpful metacognitive beliefs are elevated in psychosis and are similar to those levels observed in emotional disorder. As such, clinical formulations may benefit from the incorporation of metacognitive factors that drive unhelpful cognitive, attentional and behavioural responses. In the S-REF model metacognitive beliefs are intrinsically linked to unhelpful thinking styles that maintain distress. Metacognitive therapy specifically targets positive and negative beliefs about thinking by evaluating and questioning metacognitive beliefs (Wells, 2009, p.51). Attention training techniques and detached mindfulness are used to manage intrusions and to help people to disengage from prolonged processing that is associated with symptom maintenance and distress (i.e. the 
cognitive attentional syndrome). A recent meta-analysis suggested that metacognitive therapy (Wells, 2000, 2009) is effective in reducing symptoms associated with anxiety and depression (Normann, van Emmerik, \& Morina, 2014). Given that similar metacognitive processes appear to be implicated in emotional disorder and psychosis there is a rationale to consider metacognitive therapy in psychosis. A recent pilot study of metacognitive therapy in psychosis indicated that the therapy was an acceptable treatment with good adherence and no adverse events (Morrison et al., 2014). Furthermore, two case studies utilising the metacognitive therapy technique of attention training (Wells, 1990) suggest the technique is useful in increasing the perceived controllability of psychotic experiences and reducing distress (Levaux, Laroi, Offerlin-Meyer, Danion, \& Van der Linden, 2011; Valmaggia, Bouman, \& Schuurman, 2007). However, the results should be interpreted with caution given the lack of randomisation, the absence of a control condition and the small sample sizes.

\section{Limitations}

The data in this meta-analysis was exclusively cross-sectional. The synthesis of cross-sectional data has limitations that prevent the possibility of establishing any causal association between the variables considered. Currently, only a small number of longitudinal studies have investigated these relationships. However, findings indicate that an increased number of unhelpful metacognitive beliefs is associated with transition to clinical psychosis in people with at risk mental state (Morrison, Bentall, et al., 2002) and higher levels of negative metacognitive beliefs predict a more severe and chronic course of illness in people with a diagnosis of psychosis (Austin et al., 2015). 
The overall number of studies included was small. Therefore, the lack of significant findings in some analyses may be due to issues of statistical power rather than there being no significant effect (i.e. type-II error). In addition, there was significant heterogeneity observed in the primary analyses. This suggests there is poor consistency in the estimated effect size across studies. For these reasons, confidence in the effect estimate is limited and the true effect may vary from the summary effect we estimated.

Confounds relating to comorbidity cannot be ruled out. For example, it is not clear whether all psychosis samples considered in the primary studies were screened for comorbid emotional disorder. Likewise, it is not clear whether all emotional disorder samples were screened for psychosis. This could reduce the sensitivity and specificity of the between-group comparisons.

Finally, the measurement of metacognition in this analysis was restricted to the Metacognitions Questionnaire and its variants. On the one hand this may be a confound that introduces a source of bias. For example, several studies in this analysis included a primary author of the model being tested (Wells) or an author of this paper (Morrison or Varese). However, the remaining data included in this metaanalysis came from a diversity of research teams from different countries. In addition, it is not uncommon for researchers involved in a specific field to conduct a review of the literature. On the other hand, restricting the measurement of metacognition to the Metacognitions Questionnaire has allowed us to test one specific and influential model of psychological disorder, and decreased methodological heterogeneity of the studies analysed. It is a common criticism of meta-analysis that researcher's often combine different types of studies, in effect "mixing apples and oranges" (Borenstein, Hedges, Higgins, \& Rothstein, 2009, p. 
379). It is therefore a potential strength of the current analysis that the measurement of metacognitive beliefs has been restricted to those most relevant to the S-Ref model. However, our quality assessment indicates that the vast majority of studies included in this review, received low ratings due to failing to meet a number of key quality criteria, which is not uncommon for studies utilising cross-sectional data.

\section{Future directions}

Given that the data in this review was exclusively cross-sectional there is clearly a need for more longitudinal studies to establish the temporal relationship between metacognition, symptoms and distress. Alternatively, experimental studies might consider methods of manipulating metacognitive beliefs and assessing subsequent experiences of psychosis and/or distress. Previous reviews (Varese \& Bentall, 2011) and subsequent empirical studies (Hill et al., 2012) found that once co-variation between different symptoms is controlled for, the apparent association between hallucinations and metacognitive beliefs is considerably reduced, leading to suggestions that metacognitive beliefs might be better understood as determinants of distress rather than specific symptoms. Further investigation utilising longitudinal or experimental methodologies will allow for more rigorous tests of the prediction that metacognitive beliefs may be a general vulnerability factor for emotional dysregulation and distress. Future research could benefit from employing independent assessment of the presence of specific symptoms and distress such as the Psychotic Symptoms Rating Scales (Haddock, McCarron, Tarrier, \& Faragher, 1999), rather than generic, single-item measures of symptom severity such as the Positive and Negative Syndrome Scale (Kay et al., 1987), which often confound dimensions such as frequency, conviction, preoccupation and distress. Finally, given that different aspects and measures of metacognition appear to share other 
important relationships with psychosis and other diagnoses (e.g. Lysaker et al., 2015) future research might also consider the transdiagnostic utility of these models.

In summary, this meta-analysis found unhelpful metacognitive beliefs are elevated in people with psychosis compared to people with no psychiatric diagnosis. In addition, people with psychosis have similar levels of unhelpful metacognitive beliefs to people with emotional disorder. This suggests that metacognitive beliefs may be associated with the presence of psychological disorder and distress in general, rather than with specific symptoms or diagnoses. These findings appear to support the basic assumptions of the Self-Regulatory Executive Function model and suggest unhelpful metacognitive beliefs may be a marker for unhelpful thinking styles that maintain symptoms across disorders. However, the extent to which this relationship is influenced by comorbid emotional disorder is unclear. Nevertheless, emotional factors (i.e. anxiety and depression) are an important factor in clinical psychosis (Freeman, Garety, Kuipers, Fowler, \& Bebbington, 2002; Freeman \& Garety, 2003) and prodromal states (Yung \& McGorry, 1996). Therefore, this limitation does not dismiss the potential involvement of metacognitive beliefs in the development and maintenance of psychosis, which should be carefully examined in future longitudinal research. 
American Psychiatric Association. (2000). Diagnostic and statistical manual of mental disorders : DSM-IV-TR. Washington, DC: American Psychiatric Association.

Austin, S., Mors, O., Nordentoft, M., Hjorthoj, C., Secher, R., Hesse, M., ... Wells, A. (2015). Schizophenia and metacognition: An investigation of course of illness and metacognitive beliefs within a first episode psychosis. Cognitive Therapy and Research, 39, 61-69.

Baker, C. A., \& Morrison, A. P. (1998). Cognitive processes in auditory hallucinations: Attributional biases and metacognition. Psychological Medicine, 28(5), 1199-1208.

Barbato, M., Penn, D., Perkins, D. O., Woods, S. W., Liu, L., \& Addington, J. (2013). Metacognitive functioning in individuals at clinical high risk for psychosis. Behavioural \& Cognitive Psychotherapy, 21, 1-9.

Borenstein, M., Hedges, L. V., Higgins, J. P., \& Rothstein, H. R. (2011). Introduction to meta-analysis: John Wiley \& Sons: Chichester, UK.

Borenstein, M., Hedges, L. V., Higgins, J. P. T., \& Rothstein, H. R. (2009). Criticisms of Meta-Analysis Introduction to Meta-Analysis (pp. 377-387): John Wiley \& Sons, Ltd.

Brett, C., Johns, L., Peters, E., \& McGuire, P. (2009). The role of metacognitive beliefs in determining the impact of anomalous experiences: A comparison of help-seeking and non-help-seeking groups of people experiencing psychotic-like anomalies. Psychological Medicine, 39(6), 939950.

Cartwright-Hatton, S., \& Wells, A. (1997). Beliefs about worry and intrusions: The meta-cognitions questionnaire and its correlates. Journal of Anxiety Disorders, 11(3), 279-296.

Cohen, J. (1988). Statistical power analysis for the social sciences: 2nd edition. Hillsdale, NJ: Erlbaum.

Davis, R. N., \& Valentier, D. P. (2000). Does metacognitive theory enhance our understanding of pathological worry and anxiety? Personality and Individual Differences, 29, 513-526.

Dominguez, M. D., Wichers, M., Lieb, R., Wittchen, H. U., \& van Os, J. (2011). Evidence that onset of clinical psychosis is an outcome of progressively more persistent subclinical psychotic experiences: an 8-year cohort study. Schizophrenia Bulletin, 37(1), 84-93.

Dragan, M., \& Dragan, W. (2014). Temperament and Anxiety: The Mediating Role of Metacognition. Journal of Psychopathology and Behavioral Assessment, 36(2), 246-254.

Fraser, J., Morrison, A., \& Wells, A. (2006). Cognitive Processes, Reasoning Biases and Persecutory Delusions: A Comparative Study. Behavioural and Cognitive Psychotherapy, 34(4), 421-435.

Freeman, D., Garety, P., Kuipers, E., Fowler, D., \& Bebbington, P. (2002). A cognitive model of persecutory delusions. British Journal of Clinical Psychology, 41(4), 331-347.

Freeman, D., \& Garety, P. A. (2003). Connecting neurosis and psychosis: the direct influence of emotion on delusions and hallucinations. Behaviour Research \& Therapy, 41(8), 923-947.

Garcia-Montes, J. M., Perez-Alvarez, M., Balbuena, C. S., Garcelan, S. P., \& Cangas, A. J. (2006). Metacognitions in patients with hallucinations and obsessive-compulsive disorder: The superstition factor. Behaviour Research and Therapy, 44(8), 1091-1104.

Garety, P., Kuipers, E., Fowler, D., Freeman, D., \& Bebbington, P. (2001). A cognitive model of the positive symptoms of psychosis. Psychological Medicine, 31, 189-195.

Haddock, G., McCarron, J., Tarrier, N., \& Faragher, E. B. (1999). Scales to measure dimensions of hallucinations and delusions: the psychotic symptom rating scales (PSYRATS). Psychological Medicine, 29(04), 879-889.

Higgins, J. P. T., \& Thompson, S. G. (2002). Quantifying heterogeneity in a meta-analysis. Statistics in medicine, 21, 1539-1558.

Hill, K., Varese, F., Jackson, M., \& Linden, D. E. (2012). The relationship between metacognitive beliefs, auditory hallucinations, and hallucination-related distress in clinical and non-clinical voice-hearers. British Journal of Clinical Psychology, 51(4), 434-447.

Hjemdal, O., Stiles, T., \& Wells, A. (2013). Automatic thoughts and meta-cognition as predictors of depressive or anxious symptoms: a prospective study of two trajectories. Scandanavian Journal of Psychology, 54(2), 59-65. 
Irak, M., \& Tosun, A. (2008). Exploring the role of metacognition in obsessive-compulsive and anxiety symptoms. Journal of Anxiety Disorders, 22, 1316-1325.

Kay, S. R., Fiszbein, A., \& Opler, L. A. (1987). The Positive and Negative Syndrome Scale (PANSS) for Schizophrenia. Schizophrenia Bulletin, 13(2), 261-276.

Laroi, F., \& Van der Linden, M. (2005). Metacognitions in proneness towards hallucinations and delusions. Behaviour Research and Therapy, 43(11), 1425-1441.

Levaux, M., Laroi, F., Offerlin-Meyer, I., Danion, J.-M., \& Van der Linden, M. (2011). The effectiveness of the attention training technique in reducing intrusive thoughts in schizophrenia: A case study. Clinical Case Studies, 10(6), 466-484.

Liberati, A., Altman, D. G., Tetzlaff, J., Mulrow, C., Gøtzsche, P. C., loannidis, J. P. A., . . Moher, D. (2009). The PRISMA statement for reporting systematic reviews and meta-analyses of studies that evaluate healthcare interventions: explanation and elaboration (Vol. 339).

Lobban, F. (1998). An investigation into the role of metacognitive beliefs in auditory hallucinations and delusion beliefs. Clin. Psy. Thesis. University of Manchester.

Lobban, F., Haddock, G., Kinderman, P., \& Wells, A. (2002). The role of metacognitive beliefs in auditory hallucinations. Personality and Individual Differences, 32(8), 1351-1363.

Lysaker, P., Dimaggio, G., Wickett-Curtis, A., Kukla, M., Luedtke, B., Vohs, J., . . Davis, L. (2015). Deficits in metacognitive capacity are related to subjective distress and heightened levels of hyperarousal symptoms in adults with posttraumatic stress disorder. Journal of Trauma and Dissociation, 16(4), 384-398.

Lysaker, P., Shea, A., Buck, K., Dimaggio, G., Nicolo, G., Procacci, M., . . Rand, K. (2010). Metacognition as a mediator of the effects of impairments in neurocognition on social function in schizophrenia spectrum disorders. Acta Psychiatrica Scandinavica, 122(5), 405413.

Lysaker, P., Vohs, J. L., Ballard, R., Fogley, R. L., Salvatore, G., Popolo, R., \& Dimaggio, G. (2013). Metacognition, self-reflection and recovery in schizophrenia. Future Neurology, 8, 103-115.

McEvoy, P. M., \& Mahoney, A. E. (2013). Intolerance of uncertainty and negative metacognitive beliefs as transdiagnostic mediators of repetitive negative thinking in a clinical sample with anxiety disorders. Journal of Anxiety Disorders, 27(2), 216-224.

Moher, D., Liberati, A., Tetzlaff, J., \& Altman, D. G. (2009). Preferred reporting items for systematic reviews and meta-analyses: the PRISMA statement. PLoS Med, 6(7), 21.

Moritz, S., Peters, M. J., Laroi, F., \& Lincoln, T. M. (2010). Metacognitive beliefs in obsessivecompulsive patients: A comparison with healthy and schizophrenia participants. Cognitive Neuropsychiatry, 15(6), 531-548.

Moritz, S., Vitzthum, F., Randjbar, S., Veckenstedt, R., \& Woodward, T. S. (2010). Detecting and defusing cognitive traps: metacognitive intervention in schizophrenia. [Review]. Current Opinion in Psychiatry, 23(6), 561-569.

Morrison, A. (2001). The interpretation of intrusions in psychosis: An integrative cognitive approach to hallucinations and delusions. Behavioural and Cognitive Psychotherapy, 29, 257-276.

Morrison, A. (2005). The beliefs about paranoia scale: Preliminary validation of a metacognitive approach to conceptualizing paranoia. Behavioural and Cognitive Psychotherapy, 33(2), 153164.

Morrison, A., Bentall, R., French, P., Walford, L., Kilcommons, A., Knight, A., . . Lewis, S. (2002). Randomised controlled trial of early detection and cognitive therapy for preventing transition to psychosis in high-risk individuals: Study design and interim analysis of transition rate and psychological risk factors. The British Journal of Psychiatry, 181(Suppl43), s78-s84.

Morrison, A., French, P., Lewis, S., Roberts, M., Raja, M., Neil, S. T., . . Bentall, R. (2006). Psychological factors in people at ultra-high risk of psychosis: comparisons with non-patients and associations with symptoms. Psychological Medicine, 36, 1395-1404.

Morrison, A., Haddock, G., \& Tarrier, N. (1995). Intrusive thoughts and auditory hallucinations: A cognitive approach. Behavioural and Cognitive Psychotherapy, 23(3), 265-280. 
Morrison, A., Pyle, M., Chapman, N., French, P., Parker, S., \& Wells, A. (2014). Metacognitive therapy in people with a schizophrenia spectrum diagnosis and medication resistant symptoms: A feasibility study. Journal of Behavior Therapy \& Experimental Psychiatry, 45, 280-284.

Morrison, A., \& Wells, A. (2003). A comparison of metacognitions in patients with hallucinations, delusions, panic disorder, and non-patient controls. Behaviour Research and Therapy, 41(2), 251-256.

Morrison, A., Wells, A., \& Nothard, S. (2000). Cognitive factors in predisposition to auditory and visual hallucinations. British Journal of Clinical Psychology, 39(1), 67-78.

Morrison, A., Wells, A., \& Nothard, S. (2002). Cognitive and emotional predictors of predisposition to hallucinations in non-patients. British Journal of Clinical Psychology, 41, 259-270.

Normann, N., van Emmerik, A. A., \& Morina, N. (2014). The efficacy of metacognitive therapy for anxiety and depression: a meta-analytic review. Depression \& Anxiety, 31(5), 402-411.

Oosterhout, B., Krabbendam, L., Smeets, G., \& van der Gaag, M. (2013). Metacognitive beliefs, beliefs about voices and affective symptoms in patients with severe auditory verbal hallucinations. British Journal of Clinical Psychology, 52(3), 235-248.

Papageorgiou, C., \& Wells, A. (2001). Metacognitive beliefs about rumination in recurrent major depression. Cognitive \& Behavioural Practice, 8, 160-164.

Papageorgiou, C., \& Wells, A. (2009). A Prospective Test of the Clinical Metacognitive Model of Rumination and Depression. International Journal of Cognitive Therapy, 2(2), 123-131.

Papaleontiou-Louca, E. (2003). The concept and instruction of metacognition. Teacher Development, $7(1), 9-30$.

Perona-Garcelan, S., Garcia-Montes, J. M., Ductor-Recuerda, M. J., Vallina-Fernandez, O., CuevasYust, C., Perez-Alvarez, M., . . . Gomez-Gomez, M. T. (2012). Relationship of metacognition, absorption, and depersonalization in patients with auditory hallucinations. British Journal of Clinical Psychology, 51(1), 100-118.

Taylor, P. J., Hutton, P., \& Wood, L. (2015). Are people at risk of psychosis also at risk of suicide and self-harm? A systematic review and meta-analysis. Psychological Medicine, 45(911-926).

Valiente, C., Prados, J. M., Gomez, D., \& Fuentenebro, F. (2012). Metacognitive beliefs and psychological well-being in paranoia and depression. Cognitive Neuropsychiatry, 17(6), 527543.

Valmaggia, L. R., Bouman, T. K., \& Schuurman, L. (2007). Attention training with auditory hallucinations: A case study. Cognitive and Behavioral Practice, 14(2), 127-133.

van Oosterhout, B., Smit, F., Krabbendam, L., Castelein, S., Staring, A. B., \& van der Gaag, M. (2016). Metacognitive training for schizophrenia spectrum patients: a meta-analysis on outcome studies. Psychol Med, 46(1), 47-57.

van Rossum, I., Dominguez, M. D., Lieb, R., Wittchen, H. U., \& van Os, J. (2011). Affective dysregulation and reality distortion: a 10 -year prospective study of their association and clinical relevance. Schizophrenia Bulletin, 37(3), 561-571.

Varese, F., Barkus, E., \& Bentall, R. (2011). Dissociative and metacognitive factors in hallucinationproneness when controlling for comorbid symptoms. Cognitive Neuropsychiatry, 16(3), 193217.

Varese, F., \& Bentall, R. (2011). The metacognitive beliefs account of hallucinatory experiences: A literature review and meta-analysis. Clinical Psychology Review, 31(5), 850-864.

Wells, A. (1990). Panic disorder in association with relaxation induced anxiety: An attention training approach to treatment. Behaviour Therapy, 21, 273-280.

Wells, A. (2000). Emotional Disorders and Metacognition: Innovative Cognitive Therapy. Chichester: John Wiley \& Sons.

Wells, A. (2009). Metacognitive Therapy for Anxiety and Depression. New York: The Guilford Press.

Wells, A., \& Cartwright-Hatton, S. (2004). A short form of the metacognitions questionnaire: properties of the MCQ-30. Behaviour Research \& Therapy, 42(4), 385-396. 
Wells, A., \& Matthews, G. (1994). Attention and emotion: A clinical perspective (Vol. 2). Hove (UK): Lawrence Erlbaum.

Wells, A., \& Matthews, G. (1996). Modelling cognitive in emotional disorder: The S-REF model. Behaviour Research \& Therapy, 34, 881-888.

Wells, A., \& Papageorgiou, C. (1998). Relationships between worry, obsessive-compulsive symptoms and metacognitive beliefs. Behaviour Research \& Therapy, 36, 899-913.

Williams, J. W., Plassman, B. L., Burke, J., Holsinger, T., \& Benjamin, S. (2010). Preventing Alzheimer's disease and cognitive decline: Agency for Healthcare Research and Quality: Rockville, MD.

World Health Organisation. (1993). The ICD-10 Classification of Mental and Behavioural Disorders. Geneva: World Health Organisation.

Yilmaz, M., Gencoz, T., \& Wells, A. (2011). The temporal precedence of metacognition in the development of anxiety and depression symptoms in the context of life-stress: A prospective study. Journal of Anxiety Disorders, 25, 389-396.

Yung, A. Y., \& McGorry, P. D. (1996). The prodromal phase of first-episode psychosis: past and current conceptualisations Schizophrenia Bulletin, 22(2), 353-370. 


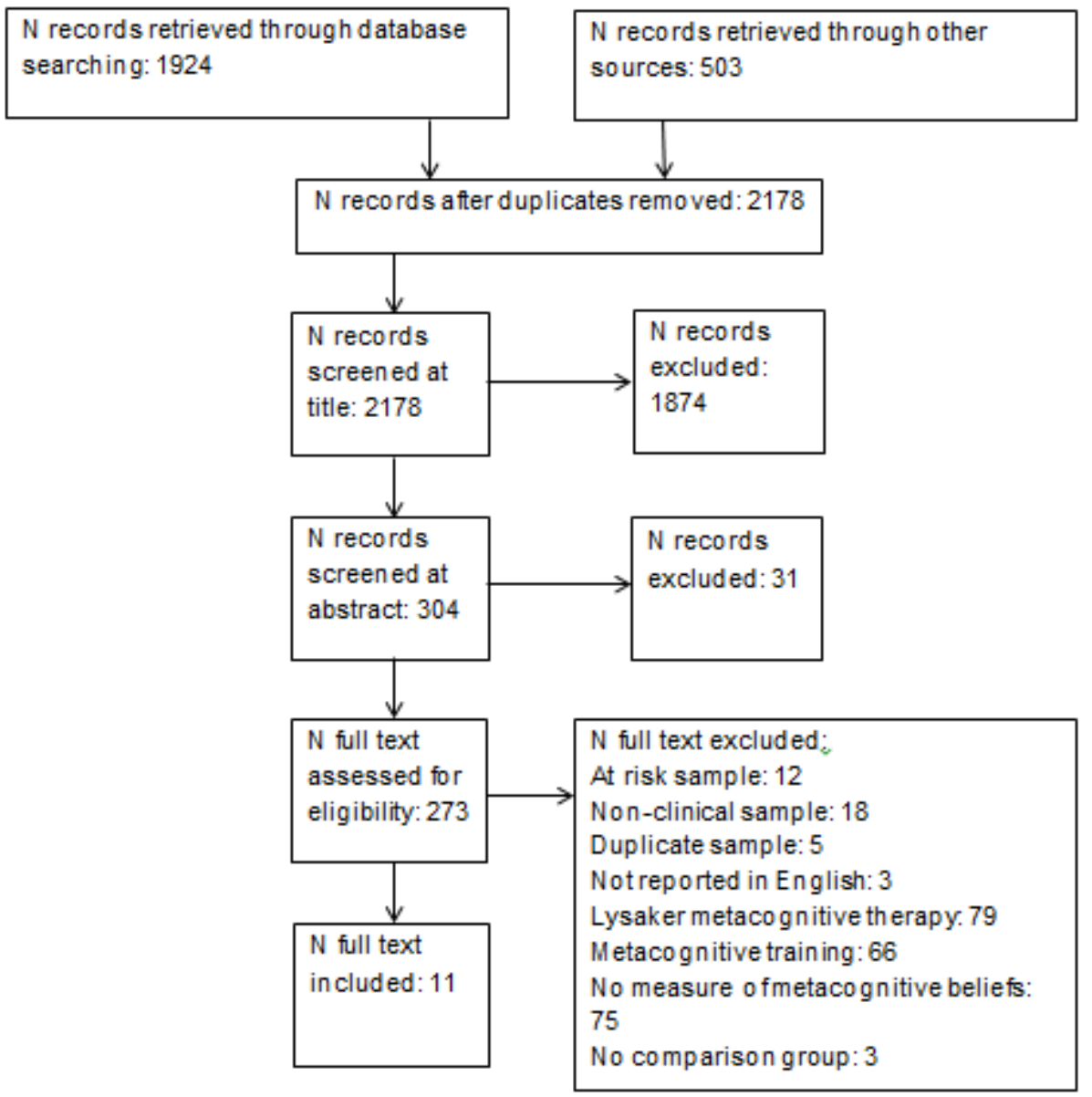

Figure 1: PRISMA diagram summarising the screening process for studies included in the meta-analysis 
Table 1: Summary of study characteristics for studies included in the statistical analyses.

\begin{tabular}{|c|c|c|c|c|c|c|}
\hline Study & Psychosis sample characteristics & $\mathrm{N}$ included & Comparator sample characteristics & $\mathrm{N}$ included & Diagnostic system & Metacognition measure \\
\hline Austin et al. (2015) & $\begin{array}{l}102 \text { psychosis } \\
25 \text { episodic psychosis } \\
214 \text { remitted psychosis }\end{array}$ & 127 & 496 non-psychiatric & 496 & ICD-10 & MCQ-30 \\
\hline Baker and Morrison (1998) & $\begin{array}{l}15 \text { psychosis with hallucinations } \\
15 \text { psychosis without hallucinations }\end{array}$ & 30 & 15 non-psychiatric & 15 & DSM-IV & MCQ-65 \\
\hline Brett et al. (2009) & 27 psychosis & 27 & 32 non-psychiatric & 32 & DSM-IV & MCQ-65 \\
\hline Fraser, Morrison, and Wells (2006) & $\begin{array}{l}15 \text { psychosis with persecutory } \\
\text { delusions }\end{array}$ & 15 & $\begin{array}{l}15 \text { Panic Disorder } \\
15 \text { non-psychiatric }\end{array}$ & $\begin{array}{l}15 \\
15\end{array}$ & DSM-IV & MCQ-65 \\
\hline $\begin{array}{l}\text { Garcia-Montes, Perez-Alvarez, } \\
\text { Balbuena, Garcelan, and Cangas } \\
(2006)\end{array}$ & $\begin{array}{l}21 \text { psychosis with hallucinations } \\
22 \text { psychosis without hallucinations } \\
16 \text { psychosis recovered from } \\
\text { hallucinations }\end{array}$ & 59 & 23 Obsessive Compulsive Disorder & 23 & DSM-IV & MCQ-65 \\
\hline Hill et al. (2012) & 20 psychosis with hallucinations & 13 & 20 non-psychiatric & 20 & PANSS & MCQ-30 \\
\hline $\begin{array}{l}\text { Lobban, Haddock, Kinderman, and } \\
\text { Wells (2002) }\end{array}$ & $\begin{array}{l}32 \text { psychosis with hallucinations } \\
23 \text { psychosis without hallucinations }\end{array}$ & 55 & $\begin{array}{l}24 \text { Anxiety } \\
28 \text { Non-psychiatric }\end{array}$ & $\begin{array}{l}24 \\
28\end{array}$ & DSM-IV & MCQ-SAM \\
\hline $\begin{array}{l}\text { Moritz, Peters, Laroi, and Lincoln } \\
\text { (2010) }\end{array}$ & 39 psychosis & 39 & $\begin{array}{l}55 \text { Obsessive-Compulsive Disorder } \\
49 \text { non-psychiatric }\end{array}$ & $\begin{array}{l}55 \\
49\end{array}$ & & MCQ-30 \\
\hline Morrison and Wells (2003) & $\begin{array}{l}49 \text { psychosis with hallucinations } \\
24 \text { psychosis with persecutory } \\
\text { delusions }\end{array}$ & 73 & $\begin{array}{l}35 \text { Panic disorder } \\
50 \text { non-psychiatric }\end{array}$ & $\begin{array}{l}35 \\
50\end{array}$ & DSM-IV & MCQ-65 \\
\hline
\end{tabular}




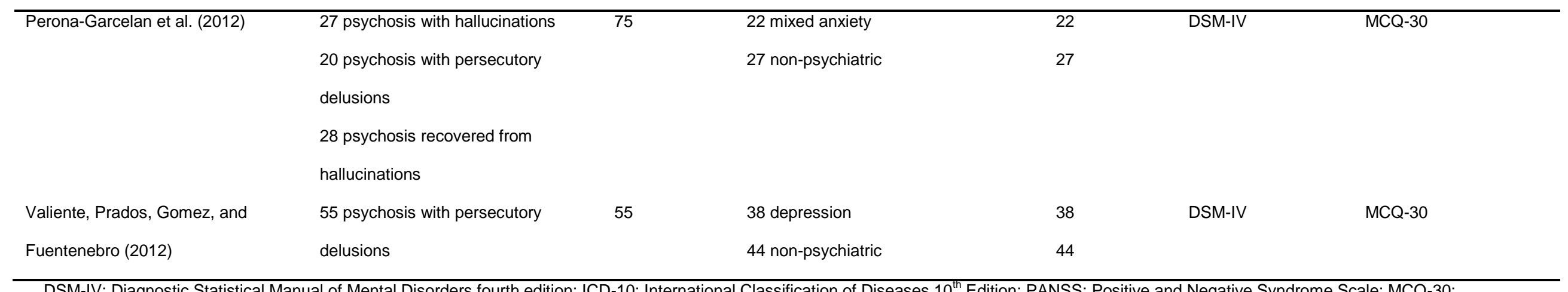

DSM-IV: Diagnostic Statistical Manual of Mental Disorders fourth edition; ICD-10: International Classification of Diseases 10 ${ }^{\text {th }}$ Edition; PANSS: Positive and Negative Syndrome Scale; MCQ-30:

Metacognitions Questionnaire-30; MCQ-65: Metacognitions Questionnaire-65 
Table 2: Overview of methodological quality assessment for studies included in the statistical analyses

\begin{tabular}{lllll}
\hline & $\begin{array}{l}\text { N studies meet } \\
\text { criteria }\end{array}$ & $\begin{array}{l}\text { N studies partially } \\
\text { meet criteria }\end{array}$ & $\begin{array}{l}\text { N studies do not } \\
\text { meet criteria }\end{array}$ & $\begin{array}{l}\text { N studies unclear } \\
\text { whether criteria is } \\
\text { met }\end{array}$ \\
\hline Unbiased selection of sample & 4 & 3 & 0 & 4 \\
Unbiased selection of control & 2 & 6 & 0 & 0 \\
Sample size calculation carried out and reported & 2 & 0 & 10 & 0 \\
Adequate description of the sample & 3 & 9 & 0 & 0 \\
Validated measure of psychosis/emotional disorder & 12 & 0 & 0 & 12 \\
Validated measure of metacognitive beliefs & 12 & 0 & 0 & 10 \\
Assessors blinded & 0 & 0 & 0 & 0 \\
Missing data reported and does not exceed $20 \%$ & 2 & 0 & 2 & 0
\end{tabular}


Figure 2: Summary effect sizes for psychosis vs. non-psychiatric control analyses

Positive Beliefs about Worry

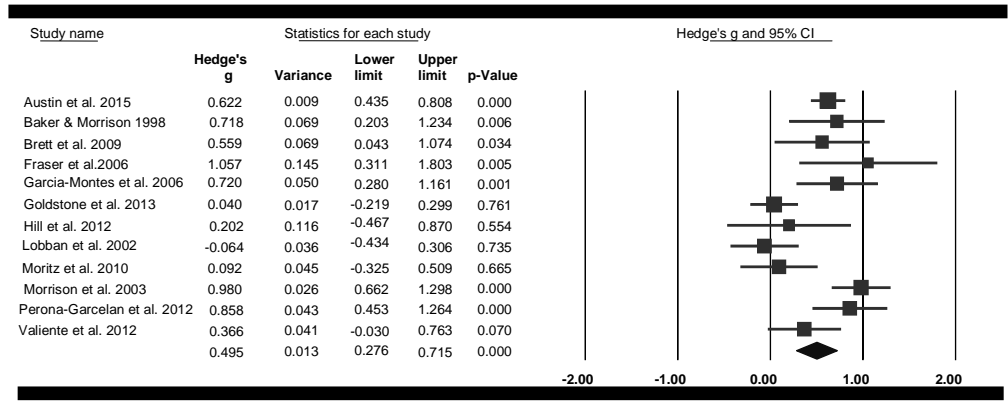

Negative Beliefs Uncontrollability and Danger

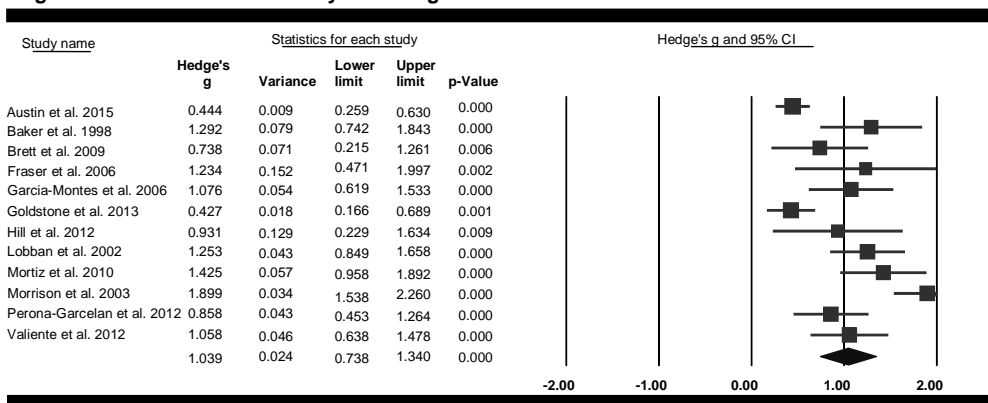

Cognitive Confidence

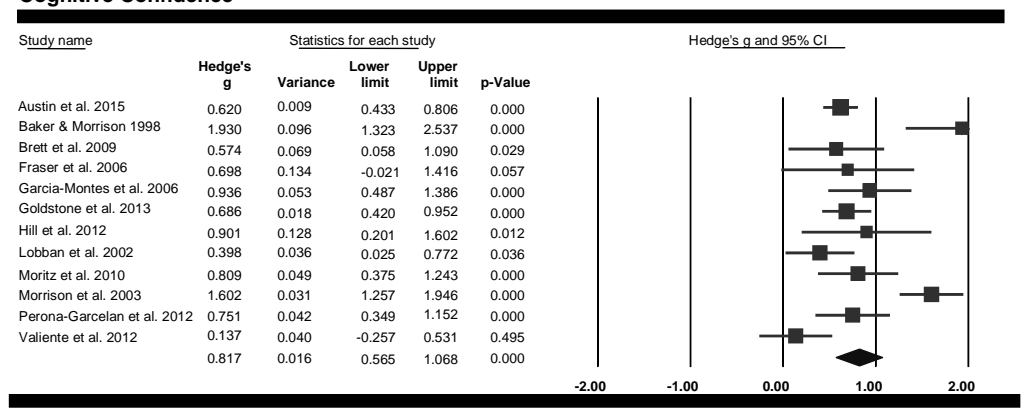

Negative Beliefs Responsibility and Superstition

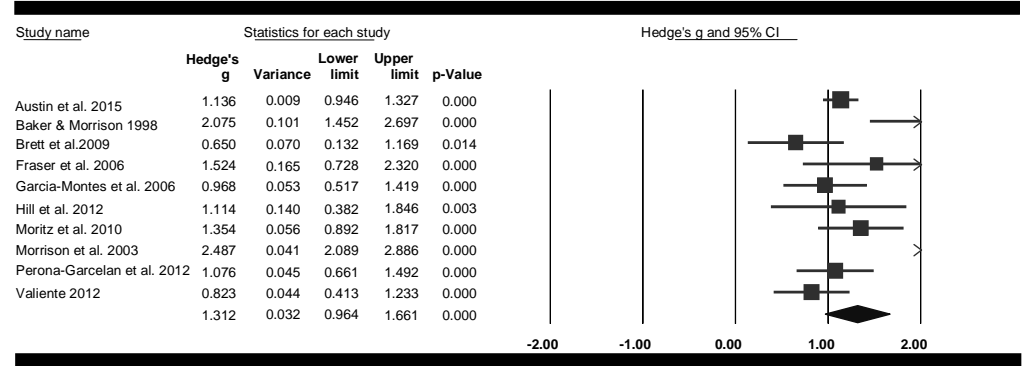

Cognitive Self-Consciousness

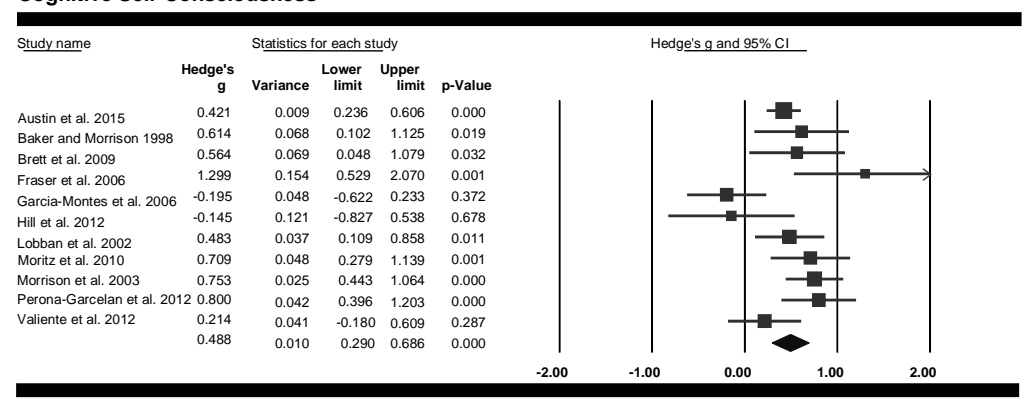


Figure 3: Summary effect sizes for psychosis vs. emotional disorder analyses

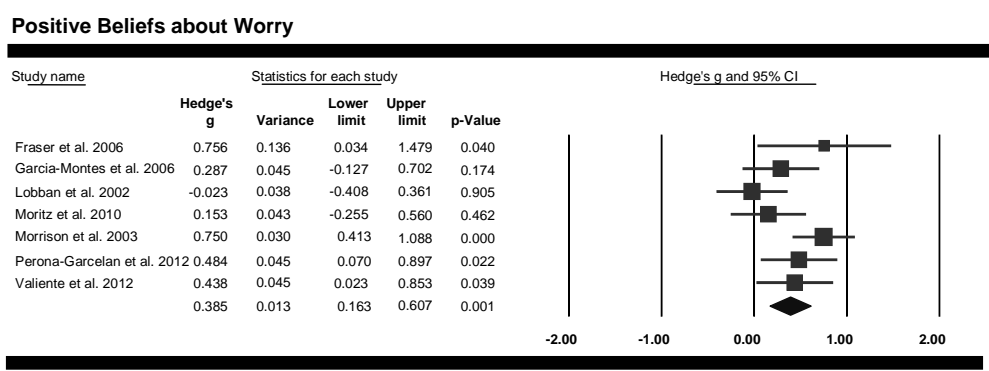

Negative Beliefs Uncontrollability and Danger

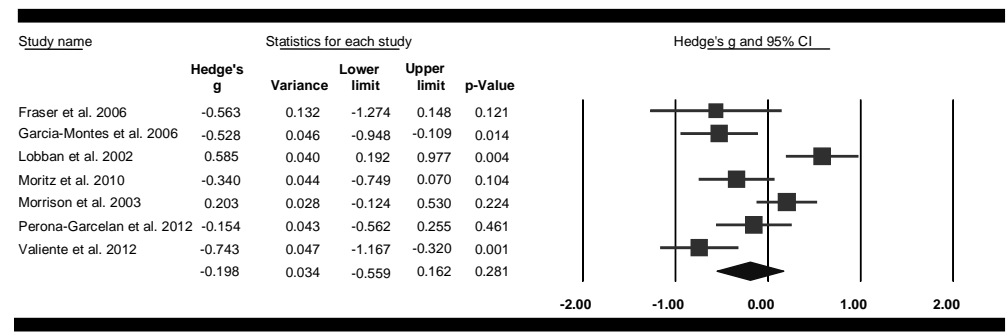

Cognitive Confidence

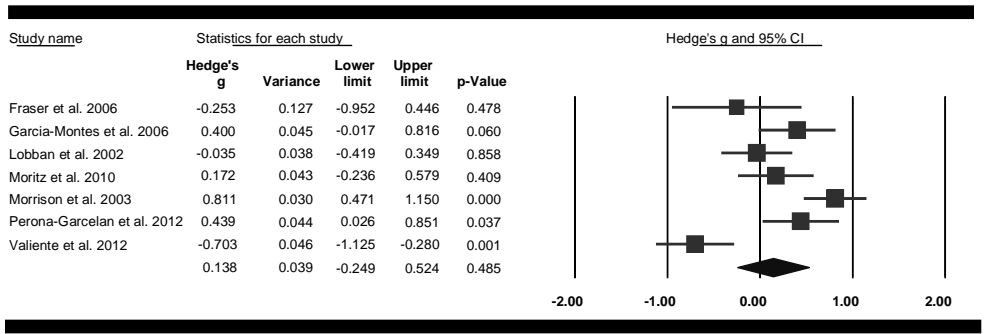

Negative Beliefs Responsibility and Superstition

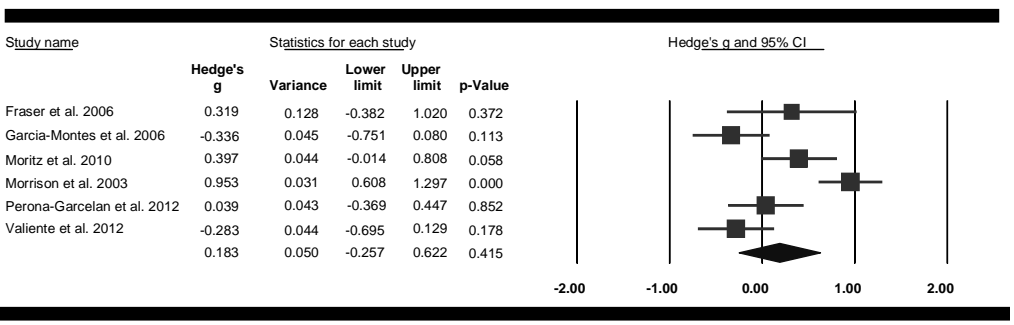

Cognitive Self-Consciousness

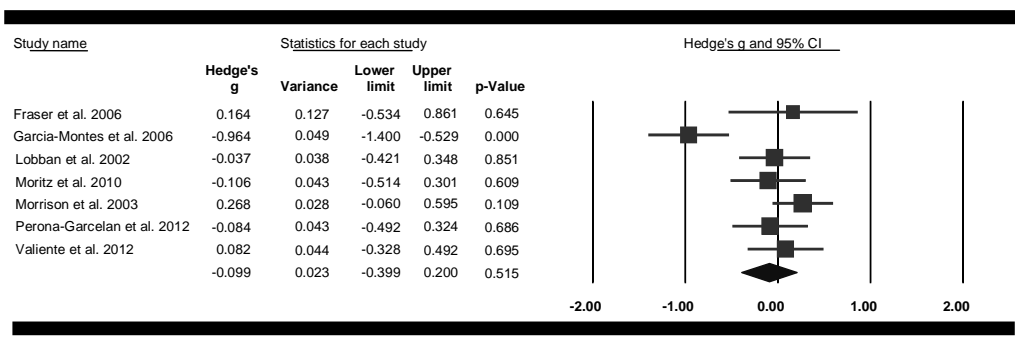


Table 3: Summary statistics for heterogeneity analyses

\begin{tabular}{llll}
\hline \multicolumn{1}{c}{$\mathrm{Q}(\mathrm{df})$} & $\mathrm{P}$ & $\mathrm{l}^{2}(\%)$ \\
\hline Psychosis and non-psychiatric control & & \\
PBW & $29.11(10)$ & 0.001 & 65.65 \\
NBUD & $64.46(10)$ & 0.000 & 84.49 \\
CC & $53.77(10)$ & 0.000 & 81.40 \\
NBRS & $57.38(9)$ & 0.000 & 84.32 \\
CSC & $26.11(10)$ & 0.004 & 61.70 \\
Psychosis and emotional disorder & & \\
PBW & $11.59(6)$ & 0.072 & 48.24 \\
NBUD & $30.67(6)$ & 0.000 & 80.44 \\
CC & $35.23(6)$ & 0.000 & 82.97 \\
NBRS & $31.56(5)$ & 0.000 & 84.16 \\
CSC & $21.28(6)$ & 0.002 & 71.80 \\
\hline
\end{tabular}

PBW: positive beliefs about worry; NBUD: negative beliefs about uncontrollability and danger; CC: cognitive confidence; NBRS: negative beliefs including responsibility and superstition; CSC: cognitive self-consciousness 